\title{
Teaching Approaches Encouraging Autonomous Learning
}

\author{
Yvan Duroc
}

Esisar, Grenoble INP, Valence, 26000, France

\begin{abstract}
This paper introduces three innovative teaching methods that encourage self-learning and self-evaluation: an original approach using recorded lessons; a new type of multiple-choice questions with embedded interactive software which offers visual applications to help answer; a method to make students more interested and involved in their training. For each method, the context and addressed pedagogical issue are described emphasizing strengths and weaknesses. The proposed approaches have been introduced for signal processing courses but they can be applied in any other fields.
\end{abstract}

Keywords Multiple-Choice Questions, Self-Learning, Self-Evaluation, Signal Processing, Video Lectures

\section{Introduction}

Autonomous learning is a process through which individuals control their own learning. Recent education theories put more emphasis on student activity than on teachers teaching. That is, they argue that the student's own experience and study are where learning starts. For example, e-learning is naturally suited to distance learning and flexible learning, and also encourages autonomous learning. The multimedia technology and Internet are become more and more pervasive and relevant pedagogical tools. The increasing tendency is the realization of new tools which use computers, partly to aid illustrations of phenomena and partly to improve efficiency in teaching processes.

In this context, the paper focuses on three innovative methods which encourage and promote self-learning and self-evaluation, and interactivity between the actors, i.e. students and instructors. The proposed approaches are the result of more than 15 years of teaching experience in signal processing (SP) with different pedagogical contexts such as trainings in engineering schools and universities, education in industrial settings and distance trainings. Although the presented methods deal with the SP field, they are general and applicable in many scientific (or not) areas.

SP courses are nowadays become not only necessary but indispensable in many engineering curricula. The teaching of SP has a long history in the field of electrical engineering (EE) which includes topics such circuit theory, electronic devices, digital electronics, microprocessors, communication systems, control systems and power electronics. With

* Corresponding author:

yvan.duroc@grenoble-inp.fr (Yvan Duroc)

Published online at http://journal.sapub.org/edu

Copyright (C) 2012 Scientific \& Academic Publishing. All Rights Reserved the development of informatics, digital signal processing (DSP) became pervasive because it is used in many important areas such as digital communication, multimedia, net working, instrumentation. DSP devices are found in many popular consumer electronic products such as MP3 players, digital camera, cell phones, modems, etc. Many areas now require a SP background, and in consequence DSP courses are taken by most students who major in electrical engineering, and by many in other engineering disciplines and scientific fields. Wherever information signal to be acquired, measured, analyzed, characterized or processed SP is present. In consequence, an enhanced SP education in the undergraduate curricula has become essential[1]-[3]. Otherwise, SP concepts are known as difficult to learn because they rely on mathematics and are not always easy to visualize. Several pedagogical approaches have been proposed to improve and facilitate SP training: use of simulation[4] or signal processors[5] to apply SP theory, interactive techniques using JAVA programs and applets to visualize SP concepts[6], interactive web platforms in order to make more accessible and interactive SP lessons[7]. All these teaching methods are relevant and present advantages to make attractive SP courses. As a result, the proposed methods that follow are not competing but complementary. According to students and instructors, level degrees, available resources, objectives of the courses, etc. they propose additional teaching materials to vary the lectures and to offer diversity.

Section II introduces in more details the context in which the SP teaching methods have been implemented. The remainder of this paper describes the three new proposed approaches. Section 3 presents an original method and its purpose using recorded lessons. Section 4 describes a new type of MCQ (Multiple-Choice Questions) integrating an interactive software which allows visual applications to help answer. Section 5 proposes an approach to make students 
more involved in their training and even becoming teachers themselves. For each method, the context and addressed pedagogical issue are described emphasizing strengths and weaknesses. Conclusion is given in section 6 .

\section{Signal Processing at Esisar}

Grenoble Institute of Technology is constituted by 6 Graduates Schools proposing 22 master's degrees in engineering. The "Electrical and Computer Engineering" (ECE) and "Computer Networks" (CN) specializations are offered in Esisar, engineering school specialized in Advanced Systems and Networks. The ECE specialization is a multi-disciplinary program which trains engineers to design, create and integrate advanced systems based upon several technological domains for embedded systems. The CN specialization trains engineers in how to design, develop and integrate complex advanced information systems and how to adapt to the evolution of languages and technology. The technological domains involved are mainly computing, networks and systems.

Considering that the student needs sound background knowledge, the first semester is common for the two specializations ECE and CN (including about 90 students). An introductory course in SP is present in the training program and represents 5 out of 30 ECTS (European Credits Transfer System). The objective of this first SP course is to tackle the fundamental concepts: Fourier analysis, continuous and discrete time signals and systems theory, random processes, and digital signal processing. With this course, students must gain knowledge of characterization and modeling of signals and systems, and how to use the associated methods and tools. As students come from various educations (French and International License Degrees, University Institutes of Technology, preparatory schools) a challenge for the instructors is to harmonize knowledge and to propose a sound course taking into account a maximum of mutual prerequisites. The success is strongly due to education in place, and above all, as a result of the personal work of students: this is why several approaches promoting self-learning have been proposed. Among the developed techniques, two approaches are noteworthy: video lectures with novel objective than classical use (section 3) and interactive customized MCQ (section 4).

Otherwise, during the last academic semester of Esisar engineering training, several advanced modules are proposed. In particular, the Engineering Control of Complex Systems (ECCS) module is specialized in Automatic Control (AC) field. The main objective of ECCS module is to present theoretical and practical tools to respond effectively to the problems of modeling, monitoring and process control of complex industrial processes (mechanical, hydraulic, thermal, etc.). In this context, the training program proposes an advanced SP course whose objectives are to complete, connect and consolidate learning in SP through practical and actual applications. The feeling of students who chose
AC module was not always good about this course because of its orientation in SP. This sensation has been inversed using a pedagogical strategy making the students involved in their training; and in the same time, the studied concepts have been seen in more-depth (section 5).

\section{New Approaches for Video Lectures}

The use of video lectures is not a new idea and has already been conducted in several universities. Some companies also offer professional solutions to achieve high quality documents. However such practices are marginal. Indeed, on the one hand, teachers are not always convinced about such investments and secondly, the technical needs a lot of resources. Nevertheless, the video lectures offers several advantages compared with traditional lectures: to present material in much less time and to be more flexible and available at no cost; once the video lectures are developed and posted. Noting that this approach alone is not sufficient to motivate students, Herman proposed a method inspired to a constructivist model of learning so-called « Just-in-time teaching »[8]-[9]. Students watched video lectures and answered online homework questions outside and then met in class to discuss their answers and practice solving problems.

The proposed approach is different and is motivated by a different objective. The initial project started from the following observation: during the course, the students do not always have time to write or understand all information provided by the teacher. Thus, when re-reading their lecture notes some parts may remain unclear. To overcome this problem, some students find as a solution to record the words of professor and listen to the difficult parts when they re-read their notes at home. This practice is marginal and the quality of recordings is unsatisfactory. In order to improve the process and to make available for all students, the following solution is set up: record lectures with a camera (audio and video) and make them available via a Web server.

The approach is absolutely different in comparison with the recording of lectures formatted for video. Students are present during lectures, and they have the opportunity to review and re-hear some misunderstood parts, i.e. have a feedback. The advantage is that the recordings (and so the lectures) are always actual. In effect the commentaries, explanations, examples, questions and answers can be different depending on the audience. Finally, although it is not the first goal, the recording can be used in case of exceptional absence and may find applications in other situations such as conferences, meetings, oral presentations, etc.

From a technical point of view, the challenge is to obtain the best possible compromise between quality and ease of implementation. Several preliminary tests have been necessary in order to define the best conditions and test the procedure. For sound, a wireless microphone provides a good quality and the teacher's speech is perfectly understandable. However students should speak loudly or a second wireless 
microphone must be used. For video, the projected documents are difficult to read but the overlay of slides solves this problem. The transparency option illustrated in Figure 1 is also interesting with an adequate.

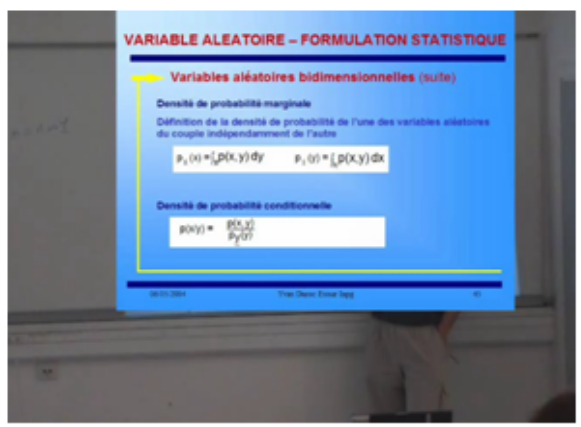

(a)

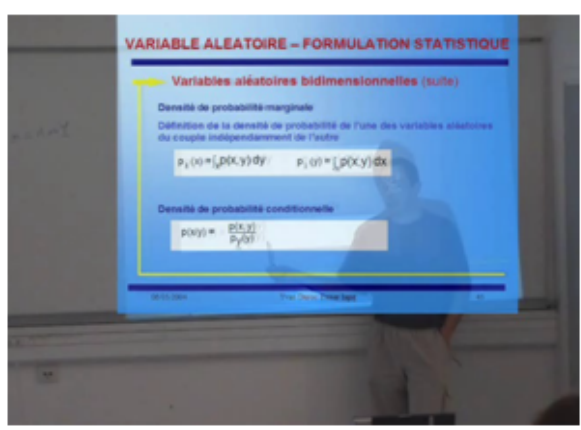

(b)

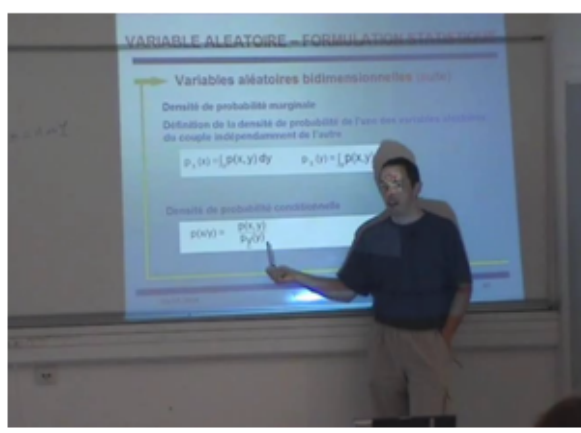

(c)

Figure 1. Video tests for how to insert the slides

Recordings are made available for download within 48 hours on a Web server. The preparation for recordings requires about ten minutes before and after the lectures. The time for the processing of video recordings, i.e. insertion of slides, compression, and upload is enough long, about 6 hours. An improvement solution would be to dispose to an equipped room and save the document directly in a format that can be put online without treatment. In order to facilitate the process, one can think that the video is not indispensable and sound recordings are sufficient. The realized experiences based on the survey of students shows that the video is a real added value making the media more attractive and pleasant to use. In effect for example, when the teacher's speech is based on a diagram or an equation, the images become more relevant. Another possible improvement is the use of graphic tablets to save the instructor's annotations. Finally, the recording procedure does not perturb the lectures, does not cause absenteeism, and implicitly, it even leads to more attention during the lectures. Statistics showed that students appreciated the modus operandi and about $60 \%$ uploaded the videos.

\section{Interactive Customized MCQ or Virtual Instructor}

MCQ is not a new method for evaluation. J. Kelly is credited with creating MCQ in 1914 at the University of Kansas. Many studies presented some keys in order to design the questions but also proposed some alternatives to improve the method[10]-[12]. The use of MCQ in educational fields is sometimes contested due to some of the negative aspects (i.e., ambiguity, no partial credit, logical reasoning, etc.), whether actual or perceived, but the format remains popular due to its utility and its advantages (i.e., efficiency, universal, neutrality, response clarity, learning the entire taught material, etc.). The SP community needs quantitative standardized tools to assess student learning in order to improve teaching methods and satisfy accreditation requirements. Thus a 25-question multiple-choice exam designed to measure students' understanding of fundamental concepts has been proposed[13]. Typical MCQ consists of two parts as follows:

i) Questions which can take the form of incomplete sentences, statements or more complex scenarios. A short text can explain the problem at hand, the setting and gives information that is relevant for solving the question, generally short yet concise.

ii) List of answer options from which one must choose either as many as true or as many as requested.

The proposed MCQ adds a third element: an interactive tool from which one can simulate the scenario concerning the questions[14]. Students can work individually their courses from a database MCQ, try to reply questions by themselves (self-evaluation) and use the joined software tool as first indication (self-learning). Using Matlab, the software providing a unique and easy to use interface is designed integrating the MCQ and embedded SP simulation tool. The toolbox Compiler converts the Matlab programs into self-contained applications which can be distributed to end users (i.e., the students) to run independently of Matlab. The stand-alone application offers the advantage to not give the possibility to access to the code and so answers' MCQ. Other computing language as the open source Scilab (scientific software package for numerical computations providing a powerful open computing environment for engineering and scientific applications) can be obviously used. Fig. 2 displays the designed interactive customized MCQ that is composed with traditional MCQ (Fig. 2a.) and associated SP tool (Fig. $2 b$.). In comparison with traditional database MCQ, the developed solution offers the possibility to switch between MCQ and a dedicated simulator. In consequence, a user can 
answer to the MCQ, and in the same time, use the SP tool in order to solve the current question or verify its solutions.

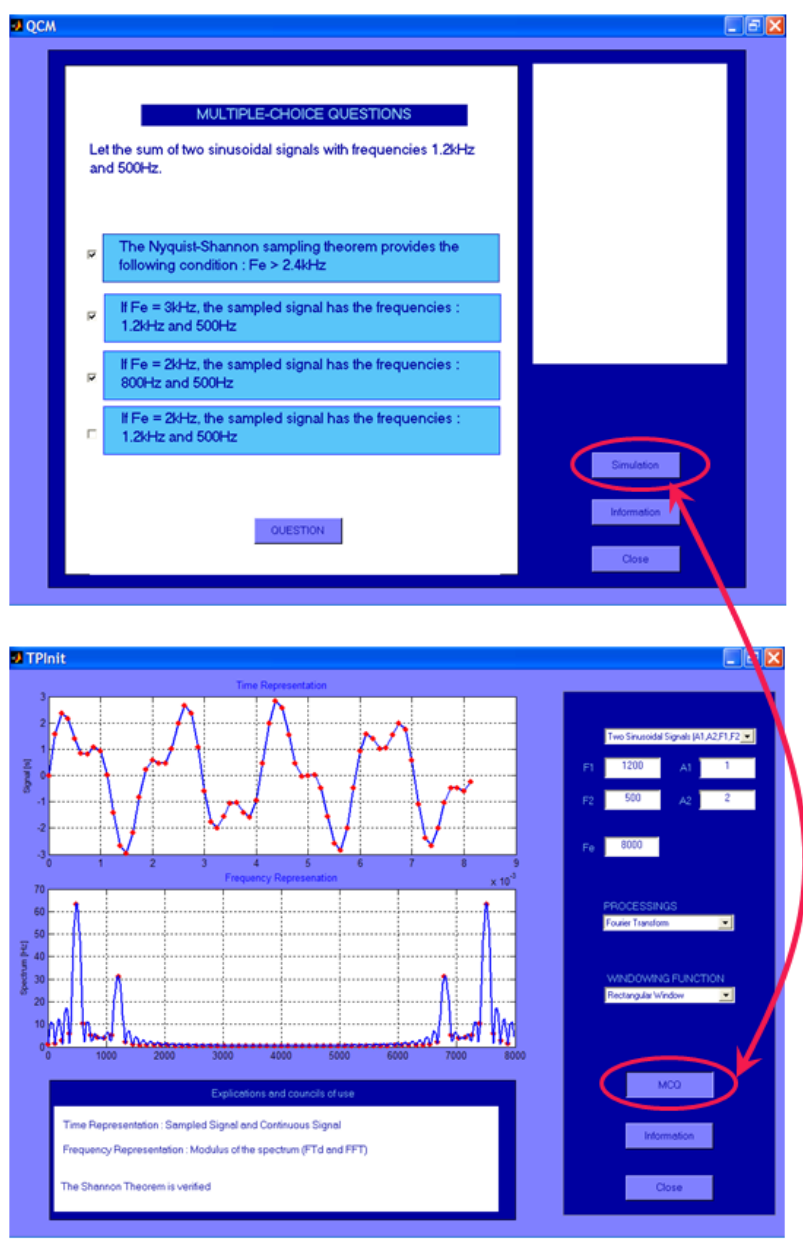

Figure 2. Interface of the customized MCQ: a) MCQ; b) SP software

The proposed customized MCQ offers a personalized pedagogical learning. The SP course can be taught in traditional methods but the students can use the described tool for understanding better the course and evaluate its knowledge. Otherwise, another possibility is to employ the customized MCQ in classroom. The instructors can then note more of participation and attention from students. Interactive resources have demonstrated that they were attractive and encouraged the students to be both active and autonomous.

\section{Students Became Teachers}

The Welsh writer Roald Dahl said "If I were a School principal, I would get rid of the History teacher and I replace it with a professor of chocolate, my students would study at least one subject that would interest them all." The approach to teach SP in the ECCS module is based on this idea.

The advanced SP course is built on some introductory lectures (about 4 hours) which are completed as follows. Groups of two to four students are constituted. Each group proposes a subject of study from given topics. If the subject is accepted by the instructor, the expected work is the fol- lowing: realize a comprehensive study in theory and simulation applications, write a report of about 20 to 25 pages, do an oral presentation (about 30-40 minutes) to the classroom. This work is part of the schedule and is based on supervised sessions: about 12 hours for preparation, and 4 hours for the presentations (in the considered cases, there are about 15 students, i.e., 5 groups). The framework is fairly strict, maintains a professional environment with accurate instructions and templates for documentation. All the realized works are shared between students. The evaluation takes into account the following criteria: autonomy, advancement reports, originality and difficulty of the topic, presentation, writing, and demonstration programs. In addition, the written test (evaluating the entire course) includes a section on all the studied and presented topics by the students.

In summary, one student has some introductory lectures, studies a topic deeply with his group, communicates on his work in writing and oral, and discovers several other topics taught by students. This type of teaching is become very popular: student motivation is enhanced, the effectiveness of learning is improved, and autonomy is developed. This approach notably demonstrates to students their own resilience to discover and take ownership of the foreground. With this approach, students have the initiative and the choice of their studied subjects. They become actors of their training. The number and the relevance of questions asked by students during the presentations also demonstrate a strong interest in all covered works. Several examples illustrating the variety of studied topics can be cited: adaptive digital filters, parametric modeling techniques, non-uniform sampling, digital communications, smart antenna systems, MUSIC technique, speech synthesis, sound synthesis methods, automatic speaker recognition system, and image processing, etc. In addition to argumentation already mentioned, there are numerous benefits: application of discovered theoretical notions, extending concepts, learning software dedicated to signal processing (Matlab / Simulink), work often going beyond the expectation (such as realization of interactive software using the Graphical User Guide of Matlab), assistance and positive mini-competition between students.

\section{Conclusion or When SP Teaching is Evaluated}

In order to promote self-learning three original methods have been presented. The principles of these methods are universal and they could be applied in other education fields than SP courses. The three proposed methods are quite different. They require a significant and progressive involvement of students in their training, from the revision of courses to the design of mini-courses. The experience showed that the interest of students for SP increased and the test scores were significantly improved.

Finally in Esisar, a systematic teaching evaluation by students is carried out for 2 years. Among the 12 common courses (in management, marketing, mathematics, computer 
science, digital architectures, network and sport) in the first semester of training program, the SP course, which is traditionally "the most difficult and feared course" was ranked first with a score of 5.4 /6 in 2011 and 5.1/6 in 2012.

\section{ACKNOWLEDGEMENTS}

The author thanks all students became "guinea-pig" during the SP courses; and more particularly, David Benaben and Jérôme Marc who helped to realize video lectures.

\section{REFERENCES}

[1] B. Boashash, S. Sridharan and V. Chandran, "A new approach to teaching signal processing at undergraduate level," in Proc. of International Symposium on Signal Processing and its Applications, Autralia, pp. 792-795, August 25-30, 1996.

[2] J.J. Zacharias and J.M. Conrad, "A survey of digital signal processing education," in Proc. of IEEE SoutheastCon, pp. 322-327, March 22-25, 2007.

[3] R.Z. Morawski, "On teaching measurement applications of digital signal processing," Elsevier Journal, Measurement, vol. 40, 2007, pp. 213-223.

[4] D. Jacoby and R. Saint-Nom, "Nice experiences teaching SP in Argentina," in Proc. of IEEE International Conference on Acoustics, Speech and Signal Processing, US, pp. 2689-2692, vol.5, May 7-11, 2001.

[5] W.S. Gan, "Teaching and Learning the hows and whys of real-time digital signal processing," IEEE Transactions on
Education, vol. 45, no. 4, November 2002, pp. 336-343.

[6] R.W. Stewart, M. Harteneck and S. Weiss, "Interactive teaching of adaptive signal processing," Engineering Science and Education Journal, vol. 9, no. 4, May 2000, pp. 161.

[7] K. Sahli, J. mars, J-L. Mari, F. Glangeaud and M. Genton, "E-learning for signal processing teaching," in Proc. of International Conference on Computer Aided Learning in Engineering Education, France, February 16-18, 2004.

[8] G.L. Herman, "Teaching signal processing according to what your students know," in Proc. of IEEE Workshop on Digital Signal Processing and Signal Processing Education, US, pp. 231-236, January 4-7, 2011.

[9] G. Novak, A. Gavrin, W. Christian and E. Patterson, "Just-in-time teaching: blending active learning with web technology", Prentice Hall, 1999.

[10] L.C., Jacobs and C.I. Chase, "Developing and using tests effectively. A guide for faculty,” Jossey-Bac Inc., 1991.

[11] R.W., Brown, "Multi-choice versus descriptive examinations," in Proc. of 31nd ASSE/IEEE Frontiers in Education Conference, US, vol.1, October 10-13, 2001.

[12] P.S., Excell, "Experiments in the use of multiple-choice examinations for Electromagnetics-related topics," IEEE Transactions on Education, vol. 43, no. 3, August 2000, pp. 250-256.

[13] K.E., Wage, J.R., Buck, C.H.G., Wright and T.B., Welch, "The signals and systems concept inventory," IEEE Transactions on Education, vol. 48, vo.3, August 2005, pp. 448-461.

[14] Y. Duroc and T.P. Vuong, "Multiple-choice question enhanced with interactive software for autonomous learning," in Proc. of IEEE International Conference on Advanced Learning Technologies, Spain, pp. 662-663, July 1-5, 2008. 